\title{
Treatment of Fourth Metatarsal Base Fracture Non-Unions in Middle Aged Women with Osteoporosis: A Case Study
}

\author{
by Lowell Weil, Jr., DPM, MBA, FACFAS ${ }^{1}{ }^{\square}$, Patrick A. McEneaney, DPM ${ }^{2}{ }^{\square}$, Jennifer L. \\ Prezioso, DPM ${ }^{3} \triangle$
}

The Foot \& Ankle Journal 1 (7): 5

Fourth metatarsal base fractures are an uncommon foot injury. These fractures can take extended periods of time to heal that is similar to a Jones fracture. The authors report on four metatarsal fractures, three of which resulted in non-unions in middle-aged women with osteoporosis. When conservative treatment failed, two cases were treated with open reduction and internal fixation while the other was treated with extracorporeal shockwave therapy. All three of these fractures ultimately led to radiographic union.

Key words: Fourth metatarsal base fracture, Jones fracture, osteoporosis, extracorporeal shockwave therapy

Accepted: June 2008 Published: July 2008

This is an Open Access article distributed under the terms of the Creative Commons Attribution License. It permits unrestricted use, distribution, and reproduction in any medium, provided the original work is properly cited. @The Foot \& Ankle Journal (www.faoj.org)

Much has been written about proximal fifth metatarsal fractures, in particular the Jones fracture. These fractures are notorious for having a slow healing potential. ${ }^{1,2}$ Because of this, more people are abdicating early fixation of the fracture with an intramedullary screw. Recently, there has been a growing concern regarding the delayed healing of fractures to the fourth metatarsal base.

Address correspondence to: Dr. Lowell Weil, Jr. DPM, MBA, FACFAS. Weil Foot \& Ankle Institute, Des Plaines, IL. 60016. Email: lwj@weil4feet.com.

\footnotetext{
${ }^{1}$ Fellowship Director, Weil Foot and Ankle Institute, Des Plaines, IL. 60016

${ }^{2}$ PGY-3, Thorek Memorial Hospital/Weil Foot \& Ankle Institute. $850 \mathrm{~W}$. Irving Park Road. Chicago, Il. 60613

${ }^{3}$ PGY-1, Thorek Memorial Hostpial/Weil Foot \& Ankle Institute. $850 \mathrm{~W}$. Irving Park Road. Chicago, IL. 60613.
}

Fourth metatarsal stress fractures commonly occur in the middle or distal diaphyseal regions. ${ }^{3}$ Metatarsal stress fractures of the fourth and fifth metatarsals comprise only $1-3 \%$ of all metatarsal stress fractures. ${ }^{4} \quad$ Proximal fourth metatarsal fractures are less common. In an epidemiological study of metatarsal fractures, Petrisor, et al., found that out of 412 metatarsal fractures, only $9 \%$ of these fractures were of the fourth metatarsal and only $2.7 \%$ were at the proximal fourth metaphyseal region. ${ }^{5}$ 
Several authors have described delayed healing and non-unions of fourth metatarsal fractures and only conservative methods were utilized in each of these cases. ${ }^{6,7,8,9}$ The authors report three metatarsal fractures that resulted in non-unions. In these three cases, the first two underwent surgical fixation of the fracture. The final case illustrates the use of extracorporeal shockwave therapy (ESWT) as an adjunct to provide adequate healing across the fracture site.

\section{Case 1}

A 47-year-old Caucasian female was evaluated for localized pain over the right fourth metatarsal after dropping a suitcase on her foot one month prior to her initial visit. She had been seen in the emergency room and diagnosed with a fracture. She was treated with a below-the-knee CAM boot and crutches.

Her past medical history included kidney and brain stem cancer. Her medications included the use of Acetaminophen/Vicodin, Spironolactone, Triamterene, and $2 \mathrm{mg}$ of Dexamethasone daily for two years. She related to being unemployed and a non-smoker. She had no history of repetitive stresses to her feet associated with exercise. Clinical evaluation revealed mild edema at the dorsal lateral aspect of the right foot and pain was elicited with palpation along the right fourth metatarsal. Plain film radiographs revealed an incomplete non-displaced lateral break of the cortex to the proximal one third of the fourth metatarsal. The patient was instructed to remain partial weightbearing in a short leg walking boot.

At one month follow up, her pain had improved slightly, but there was no evidence of healing across the previously noted fracture site of the fourth metatarsal. She was kept partial weightbearing with the short leg walking boot. A combined magnetic field bone stimulator (DonJoy®, Vista, California) was also utilized three months post injury.
When the patient returned at four months post injury, the pain had not improved and a radiographic non-union with slightly displacement was observed.

After one more month in a stiff soled shoe and an accommodative insert, no healing across the fracture site was evident on CT scan. Therefore, the decision was made to utilize surgical intervention. The fracture site was cleaned of any debris and the bone ends were drilled to promote bleeding. Trinity ${ }^{\mathrm{TM}}$ Multipotential Cellular Bone Matrix (Blackstone Medical Inc., Springfield, MA) was placed in the fracture site and a Stryker four hole locking plate (Stryker, Kalamazoo, MI) was placed in a dorsal fashion over the fourth metatarsal. The patient was placed nonweightbearing in a below-the-knee cast.

After two weeks, the patient had minimal pain and, radiographically, the fracture was in normal anatomical alignment. She was allowed partial weightbearing in a short leg, below-the-knee boot for the next four weeks. During this time, she began physical therapy for range of motion, strengthening, and gait training. She healed uneventfully.

\section{Case 2}

A 67-year-old Caucasian female presented ten days following an inversion type ankle sprain. She had localized pain over the right fourth metatarsal. The patient admitted to being diagnosed with a stress fracture of her right foot approximately seven months prior for which she was instructed to ambulate with the use of a surgical shoe. Her past medical history included osteoporosis. Her present medications include Ibandronate, Amitripyline, Raloxifene, and Zolmitriptan. She is a non-smoker who worked part time as a teacher's aid. Radiographs revealed a fracture to the right fourth metatarsal. She did not return to normal shoe gear until five months following the initial diagnosis. 


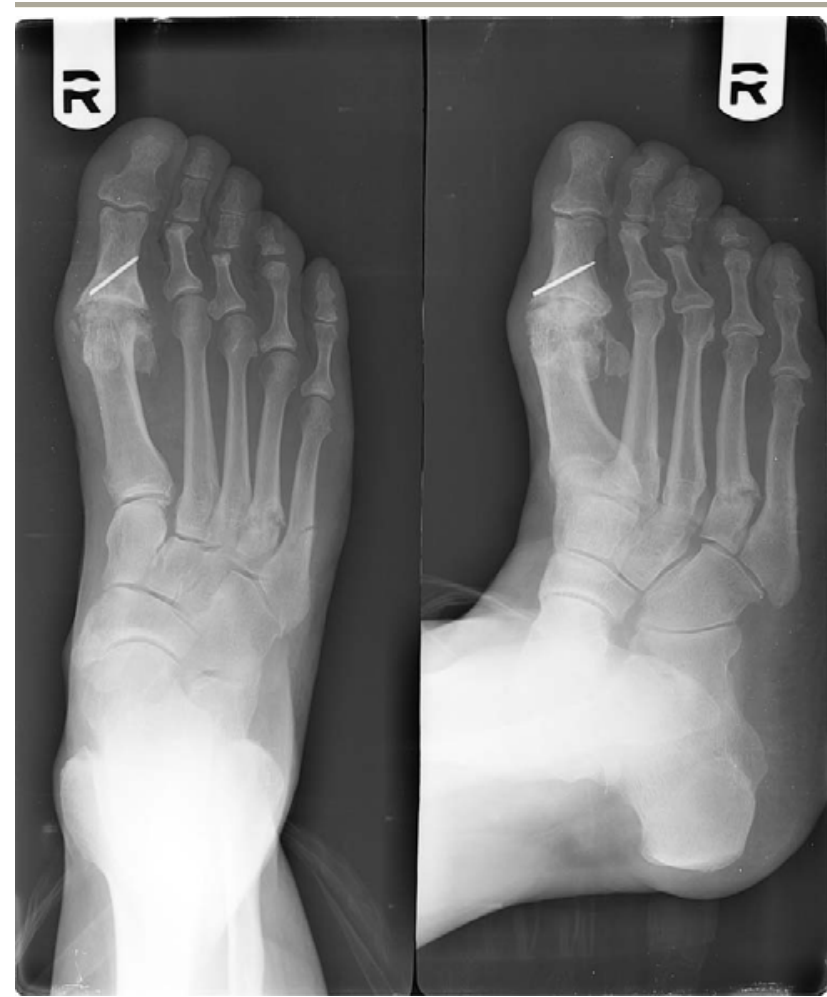

Figure 1 Plain film radiographs of the right foot display a complete non-displaced fracture of the proximal fourth metatarsal with mild gapping. A non-displaced fifth metatarsal fracture is also seen.

There was significant edema over the dorsal lateral aspect of the right foot with pain noted along the fourth metatarsal, especially at the base. Plain film radiographs of the right foot revealed a complete non-displaced fracture of the proximal fourth metatarsal with mild gapping.

There was no callus formation across the fracture site. She was advised to be partial weightbearing in a short leg walking boot. After four weeks, her symptoms did not improve. Radiographs of the right foot were taken revealing no evidence of healing across the fracture site of the fourth metatarsal base. A new fracture at the diaphysis of the fifth metatarsal was noted. (Fig. 1) The patient was instructed to continue partial weightbearing in the below-the-knee walking boot.

After five more weeks, there were still no signs of healing at the fourth metatarsal, and there was an incomplete midshaft fracture at the fifth metatarsal.

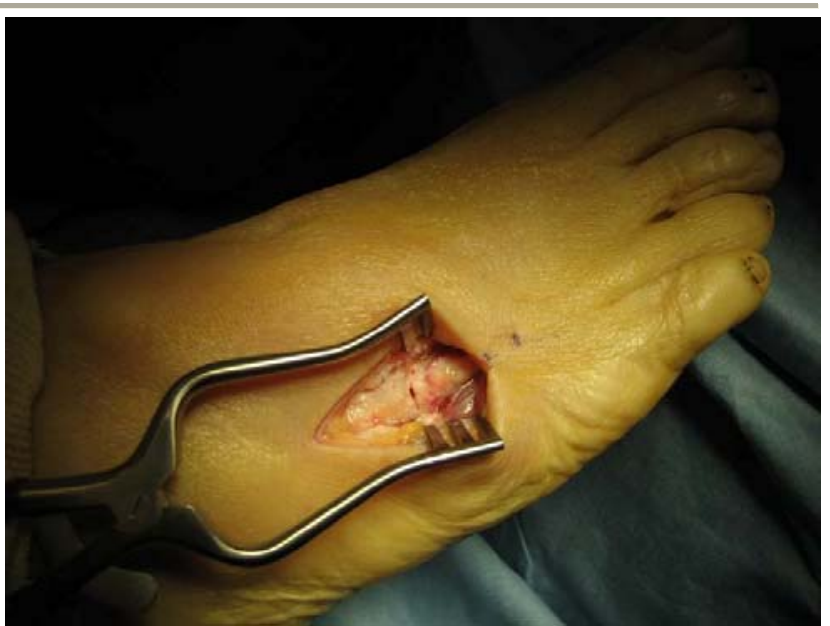

Figure 2 Intra-operative picture of fracture non-union.

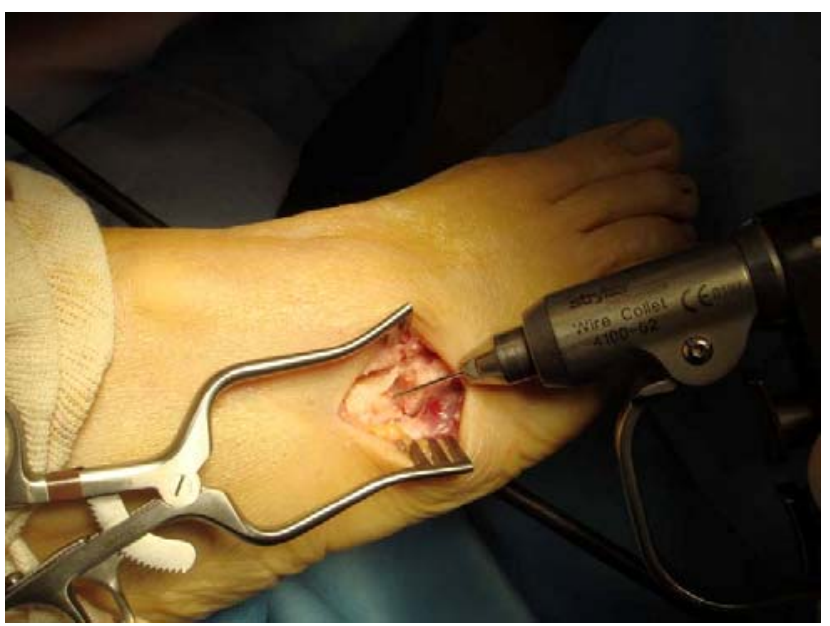

Figure 3 Intra-operative picture of drilling the fractured ends.

Since the fourth metatarsal fracture showed no evidence of healing, the patient elected for open reduction internal fixation of the fourth right metatarsal. A dorsal approach was performed to access the fourth metatarsal base. (Fig. 2)

The fracture site was cleaned of any debris, and the bone ends were drilled to promote bleeding. (Fig. 3).

Trinity $^{\mathrm{TM}}$ Multipotential Cellular Bone Matrix was placed in the fracture site (Fig.4) and a Stryker ${ }^{\circledR}$ four hole locking plate was placed in a dorsal fashion over the fourth metatarsal (Fig. 5) 


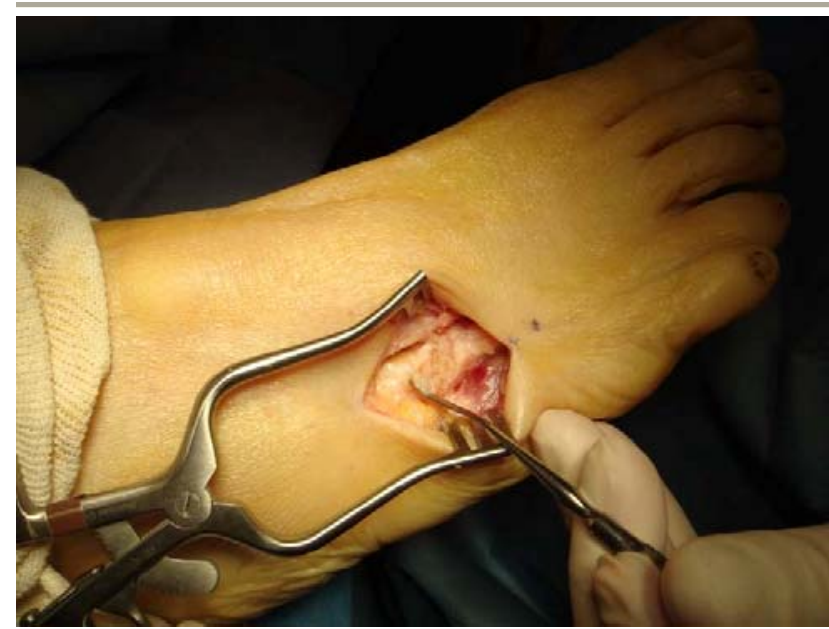

Figure 4 The fracture defect is packed with Trinity $^{T m}$ Multipotential Cellular Bone Matrix.

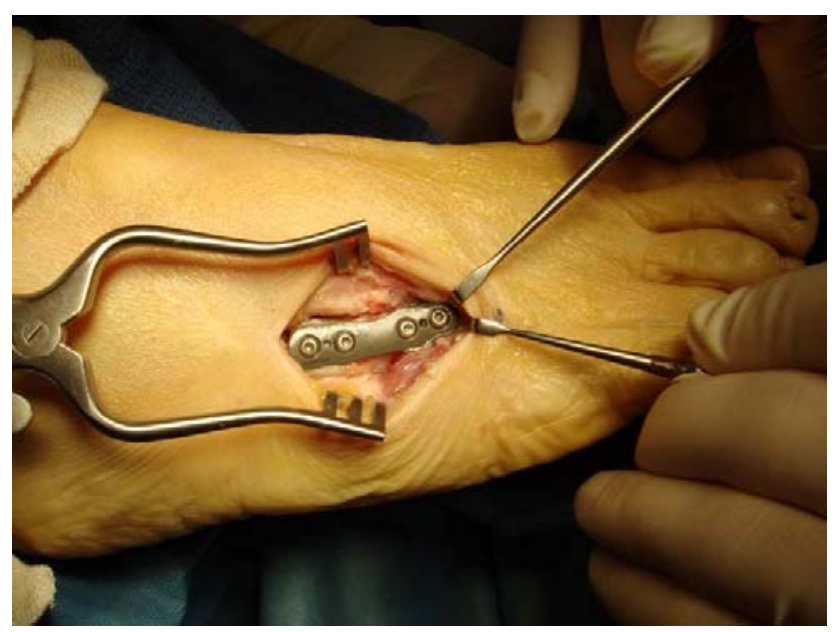

Figure 5 A Stryker ${ }^{\circledR}$ Locking plate is used to bridge the fracture site.

She was placed non-weightbearing in a below the knee cast and healed uneventfully in six weeks time.

After 2 months, the fracture site revealed good bone consolidation and healing. (Fig. 6)

\section{Case 3}

A 46-year-old Caucasian female presented with a chief complaint of lateral metatarsal pain to both feet. She was an avid runner with a history of stress fractures over the previous four years.

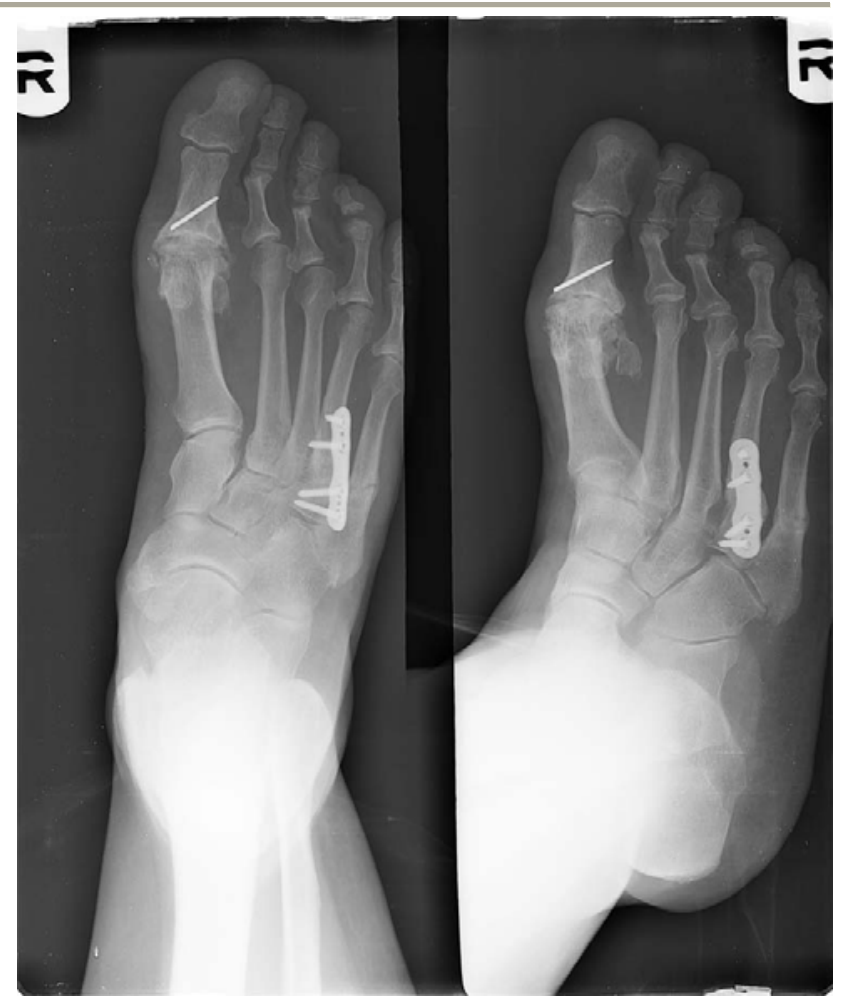

Figure 6 Radiographs show good bone consolidation and healing of the fourth metatarsal fracture site 2 months after surgery.

Her most recent stress fracture was at the left fourth metatarsal diagnosed two months prior to her initial presentation. She had been placed partial weightbearing in a boot for one month and was later transitioned into orthotic devices and her running shoes. The right foot had been pain free while there was moderate pain of the left foot.

Her past medical history was remarkable for osteoporosis and acid reflux. The patient's current medications included Risedronate, Lansoprazole, and Norethindrone/Ethinyl Estradiol. She is a non-smoker. There is localized tenderness over the fourth and fifth metatarsal bases of the right foot and no edema was seen over the area. Biomechanical examination revealed equinovarus and a severe metatarus adductus deformity bilaterally. A bone stimulator was placed on the foot for use thirty minutes a day. She was instructed to refrain from running. 


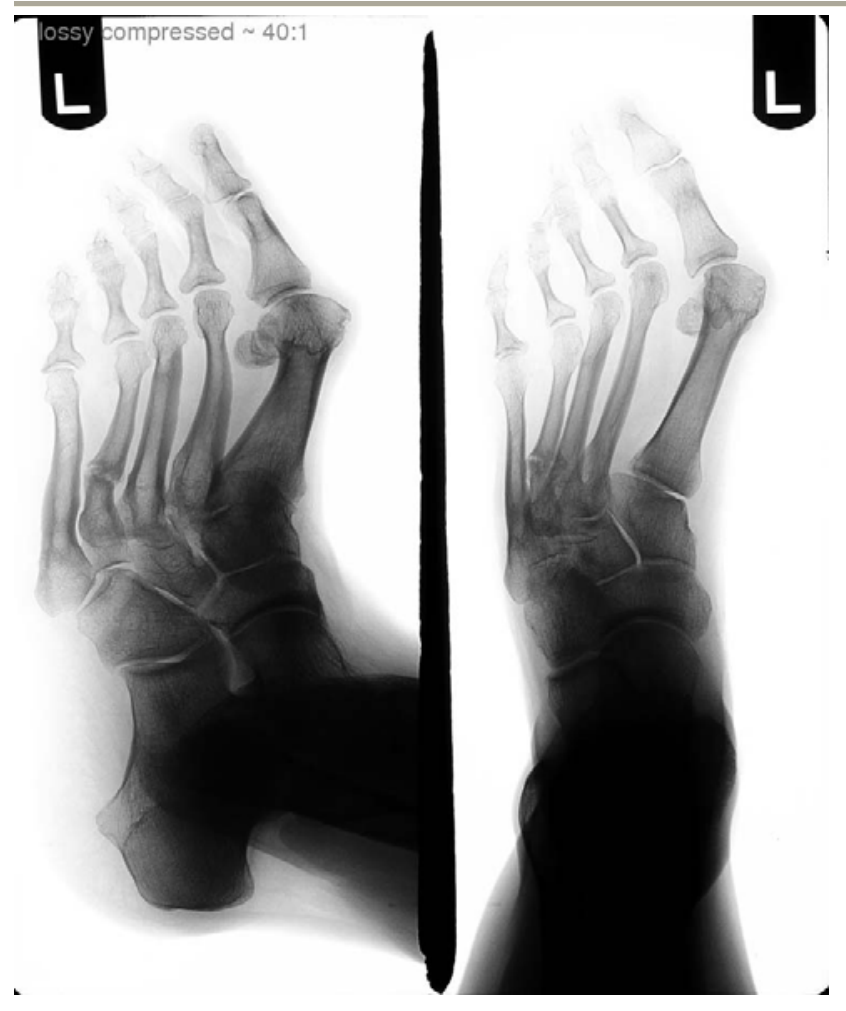

Figure 7 Fourth metatarsal fracture of case \# 3 (left foot only) showing painful, hypertrophic non-union.

She was seen one month later with report of no improvement in her symptoms. Plain film radiographs revealed evidence of new bone callus formation across the fifth metatarsal fracture sites of the right, and no change of the left fourth metatarsal fracture. She was instructed to be partial weightbearing in a surgical shoe on the left and to continue to limit her activities. Five months post injury, her pain had slightly improved, and she was still quite tender to palpation over the fourth metatarsal. Radiographs of the left foot revealed hypertrophic nonunion of the fourth metatarsal, while the right foot fracture demonstrated signs of osseous bridging. (Fig. 7)

She expressed no interested in surgery; therefore she was treated with high energy ESW'T followed by a period of partial weightbearing in a walking boot. The patient received ESWT with Intavenous Anesthesia. A Orbasone ${ }^{\text {TM }}$ electrohydraulic device was utilized at $22 \mathrm{Kv}$ for 4000 shocks directly over the fracture/nonunion site.

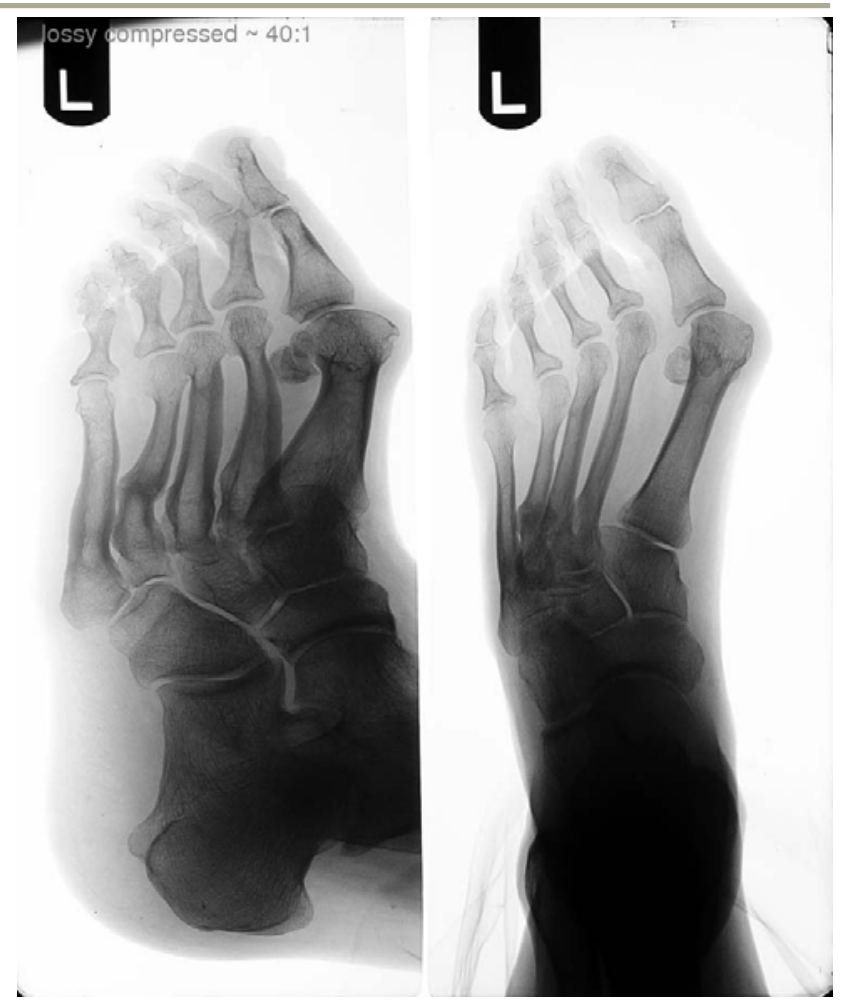

Figure 8 Fourth metatarsal fracture of case \# 3 after a single treatment of ESWT.

At one month follow up, her pain had completely resolved, and there was minimal localized tenderness upon palpation of the fracture site. Radiographs revealed some consolidation across the fracture site. (Fig. 8) The patient was allowed to begin light exercise with avoidance of high impact activity. Three months following ESWT, the patient had no tenderness to palpation of the left fourth metatarsal. Radiographs showed continued consolidation of the fracture site.

\section{Discussion}

Hetsroni, et al., theorized that proximal fourth metatarsal fractures may be related to anatomic, functional, and vascular mechanism similar to the Jones fracture. ${ }^{6}$ The nutrient artery to the fourth metatarsal enters on the lateral side, while the fifth metatarsal nutrient artery enters on the medial side. ${ }^{10}$ Due to their close anatomical relationship, both arteries are subject to similar biomechanical forces that can result in injury. 
The fourth and fifth metatarsals perform similar actions by allowing for adduction and plantarflexion of the foot. Therefore, abnormal biomechanical forces can traverse both metatarsals.

Theodorou, et al., reported six patients with metatarsal stress fractures associated with an adducted forefoot.' These patients did not have the history of repetitive impact activity, but some of the patients had a history of poor bone stock, a neuropathic foot, or multiple stress fractures. It was found that $77 \%$ were in a transverse pattern and $86 \%$ were located in the proximal one third. The patients were kept non-weightbearing or partial weightbearing with an orthotic device and healed without complication in four to eight weeks. The third case of fourth metatarsal fracture occurred in a severely adducted forefoot while there was a mild metatarsus adductus in the first case. These factors may have also contributed to the occurrence of other fractures.

In a series of five fourth metatarsal fractures in active people, Saxena, et al., found that the healing time of these injuries was eight to sixteen weeks. ${ }^{8}$ They also found that patients who did not go through the recommended period of immobilization took up to eight months to return to normal activity. Their recommendation is nonweightbearing with below-the-knee cast immobilization or walking boot.

Shearer and Penner reported two stress fractures of the fourth metatarsal bases that occurred in healthy patients with normal bone stock and without an adducted forefoot, but a mild pes planus. ${ }^{7}$ These patients were treated with belowthe-knee cast immobilization for eight weeks followed by gradual return to activities and orthoses. ${ }^{7}$ After cast removal, they recommended that activities should be gradually resumed and appropriate orthoses considered. The authors concluded that final evaluation of the fracture healing should be based on clinical findings and the CT scan because radiographs may not demonstrate bony union.
In this series, after up to six months of conservative care, there was little evidence of radiographic healing and the patients were still symptomatic. In the first two cases, surgical intervention achieved satisfactory results.

Locking plate technology was used to create stability across the fracture sites. Trinity ${ }^{\mathrm{TM}}$ Multipotential Cellular Bone Matrix was placed in the fracture site to provide a rich source of stem cells with osteogenic, osteoinductive and osteoconductive properties to aid in bone healing.

In the third case, high energy ESW'T was utilized to facilitate healing of the fracture site. High energy ESWT has been shown to promote fracture healing and have a lower non-union rate versus control groups. ${ }^{11}$ Bara and Snyder found ESWT to be an effective treatment for delayed union or non-union of bone with a union rate of $83 \%$ after three to six months. ${ }^{12}$ In a study by Wang, et al., the results of shockwave treatment were similar to the results of surgical treatment for chronic non-unions. ${ }^{13}$

In this case series, all patients were middle-aged postmenopausal women. The fracture in the first case was caused by direct trauma and most likely resulted in a non-union secondary to long term oral corticosteroid use. Corticosteroids are well known to cause osteoporosis and increase the risk of pathological fracture. ${ }^{14,15,16}$ In all three of these cases, decreased bone mineral density may have been a contributing factor. While objective bone density values were not identified in these two patients, the patients were being treated with bisphosphonates for osteoporosis.

All patients were partial weightbearing in a belowthe-knee pneumatic walking boot. In all reports, patients were either instructed to be nonweightbearing in a below-the-knee cast/boot ${ }^{7,8.9}$ or partial weightbearing in an orthotic device. ${ }^{9}$ All fractures resulted in union. It is unclear whether these fractures would have healed if the patients were kept non-weightbearing. 


\section{Conclusion}

The authors believe fourth metatarsal base fractures, like fifth metatarsal fractures (Jones fracture), have a tendency towards prolonged healing and non-union.

Other factors such as decreased bone mineral density and long term steroid therapy may also contribute to non-union. While most fractures will heal with extended conservative care, surgical intervention is often necessary in patients with painful non-unions. Extracorporeal shockwave therapy can be considered in those patients with delayed unions or in patients with non-unions that are not interested in surgery. The authors feel that early surgical intervention may result in decreased disability and earlier return to normal activity.

\section{References}

1. O'Shea, M. et al Clinical Perspective of the Treatment of Fifth Metatarsal Fractures. J Am Podiatr Med Assoc. 85 (9): 473-480, Sep 1995.

2. Holmes, G. et al Treatment of Delayed Unions and Nonunions of the Proximal Fifth Metatarsal with Pulsed

Electromagnetic Fields. Foot Ankle Int. 15 (10): 552-556, Oct 1994.

3. Meurman, K. Less common stress fractures in the foot. Br J Radiol. 54(637): 1-7. Jan 1981.

4. Levy, J. Stress fractures of the first metatarsal. AJR Am J Roentgenol. 130(4): 679-81. Apr 1978.

5. Petrisor, B. et al The Epidemiology of Metatarsal Fractures. Foot Ankle Int. 27 (3): 172-174, Mar 2006.

6. Hetsroni, I. et al Base of Fourth Metatarsal Stress Fracture: Tendency for Prolonged Healing. Clin J Sport Med. 15 (3): 186188, May 2005.

7. Shearer, C. et al Stress Fractures of the Base of the Fourth Metatarsal: 2 Cases and a Review of

the Literature. Am J Sports Med. 35 (3): 479-483, Mar 2007.

8. Saxena, A. et al Proximal Fourth Metatarsal Injuries in Athletes: Similarity to Proximal Fifth Metatarsal Injury. Foot Ankle Int. 22 (7): 603-608, Jul 2001.

9. Theodorou, D. et al Stress Fractures of the Lateral Metatarsal Bones in Metatarsus Adductus Foot

Deformity: A Previously Unrecognized Association. Skeletal Radiol. 28 (12): 679-684, Dec 1999.

10. Shereff, M. et al Vascular Anatomy of the Fifth Metatarsal. Foot Ankle. 11 (6): 350-353, Jun 1991.

11. Wang, C. et al The Effects of Extracorporeal Shockwave on Acute High-Energy Long Bone Fractures of the Lower Extremity. Arch Orthop Trauma Surg. 127 (2): 137-142, Feb 2007.

12. Bara, T. et al Nine Years Experience with the Use of Shock Waves for Treatment of Bone Union Disturbances. Ortop Traumatol Rehabil. 9 (3): 254-258, May-Jun 2007.

13. Wang, C. et al Treatment of Non-unions of Long Bone Fractures with Shock Waves. Clin Orthop Relat Res. 387: 95-101, Jun 2001

14. Adachi, J. Corticosteroid-Induced Osteoporosis. Am J Med Sci. 313 (1): 41-49 Jan 1997.

15. Eastell, R. et al A UK Consensus Group on Management of Glucocorticoid-Induced Osteoporosis: An Update. J Intern Med. 244 (4): 271-292, Oct 1998

16. Van Staa, T. et al Use of Oral Corticosteroids and Risk of Fractures. J Bone Miner Res. 20 (8): 1487-1494, Aug 2005. 